\title{
Einführung: Allgemeine Grundlagen der gastrointestinalen Motilitåt
}

\author{
Hans-Dieter Allescher
}

\section{Allgemeiner Aufbau des Gastrointestinaltraktes}

Der Magen-Darm-Trakt des Menschen besteht bis auf das obere Drittel der Speiseröhre und den Sphinkter ani externus aus glatter Muskulatur, die ontogenetisch dem Entoderm entstammt. Die Innervation dieser glatten Muskulatur erfolgt durch eine komplexe nervale Struktur, das enterische Nervensystem, das als eigenständige autonome Schaltzentrale für die Regulation von Motilität, Sekretion und Durchblutung verantwortlich ist [1].

Die extrinsischen autonomen Nerven des Gastrointestinaltraktes, der N. vagus, die parasympathischen Anteile des sakralen Rückenmarks und die sympathischen Nervenfasern haben zwar auch eine modulierende Funktion, besitzen aber im Wesentlichen eine afferente Funktion, was daran erkennbar ist, dass die vagalen und sympathischen Nerven überwiegend aus afferenten Nervenfasern bestehen.

\section{Grundlagen der Motilitåtsphånomene}

Grundsätzlich lassen sich verschiedene Formen der gastrointestinalen Motilität beschreiben. Die Darmwand ist zu Kontraktionen fähig, die durch exzitatorische Innervation oder durch Reduktion der hemmenden Innervation ausgelöst werden [2]. Die glatte Muskulatur kontrahiert sich dabei nicht wie die quergestreifte Muskulatur lediglich in Abhängigkeit von den nervalen Impulsen, vielmehr wird der Kontraktionsrhythmus von periodisch auftretenden Depolarisationen der glatten Muskulatur vorgegeben [3]. Diese langsamen Wellen sind in ihrer Frequenz für die einzelnen Darmabschnitte stabil und spezifisch und werden durch ein weiteres spezialisiertes Zellsystem, die interstitiellen Cajal-Zellen, erzeugt [4].

Die koordinierten propulsiven Kontraktionen des Darmes werden durch einen weiteren grundlegenden Basisreflex, den peristaltischen Reflex, ermöglicht. Dieser von Balis und Stärling 1899 beschriebene Reflex bewirkt, dass sich ein Darmabschnitt proximal einer Dehnung kontrahiert und distal (aboral) davon reagiert [5], [6]. Er stellt den wesentlichen Grundbaustein für eine geordnete peristaltische Aktivität im Gastrointestinaltrakt dar.

Im oberen Gastrointestinaltrakt lassen sich ab der Magenregion zwei weitere grundlegende Motilitätsmuster nachweisen:

- die interdigestive Motilität im Nüchternzustand („Nüchternmotilität“),

- das postprandiale Motilitätsmuster („digestive Motilität“).

Das interdigestive Motilitätsmuster ist dadurch gekennzeichnet, dass charakteristische, zyklische Motilitätsmuster von proximal (Magen, Dünndarm) nach distal ab- 
laufen (Jejunum, Ileum). Die einzelnen Phasen dieses Zyklus unterscheiden sich durch ihre Kontraktionsaktivität und lassen sich nach ihrem Kontraktionsmuster in drei Phasen einteilen [7],[8] (s. auch Abschn. 5 der Einführung).

Die interdigestive Motilität kann durch Aufnahme (Kauen, Schlucken) einer Mahlzeit oder aber auch durch zephale Stimulation unterbrochen werden, sodass im gesamten oberen Gastrointestinaltrakt (Magen und Dünndarm) eine gleichförmige kontinuierliche motorische Aktivität, die digestive Motilität, einsetzt [9].

Neben diesen physiologischerweise auftretenden Motilitätsmustern existieren noch weitere spezifische pathophysiologische Motilitätsmuster, die einem vorprogrammierten, koordinierten Ablauf folgen. Dies sind z. B. die retropulsive Motilität beim Erbrechen oder die stark propulsiven Kontraktionen im Dickdarm (,giant migrating contractions") bei einigen Formen der Diarrhöe. Am oralen Beginn des Gastrointestinaltraktes (Pharynx, Ösophagus) und am distalen Ende (Rektum, Anus), wo die autonome koordinierte Tätigkeit in den Willkürbereich des Schluckens bzw. der Defäkation übergeht, sind spezielle Motilitätsmuster vorhanden, die eine Koordination mit den gesteuerten Willkürprozessen der quergestreiften Muskulatur ermöglichen.

\section{Grundlagen der Muskelfunktion}

\subsection{Elektrophysiologie und Funktion der glatten Muskulatur}

Die glatte Muskulatur des Darmes ist durch spezifische myoelektrische Aktivitätsmuster gekennzeichnet. Es lassen sich typische basale und stimulierte myoelektrische Aktivitäten unterscheiden.

Die basalen, rhythmischen Depolarisationswellen, sog. langsame Wellen oder „slow waves“, sind periodische, wellenförmige Depolarisationen der glatten Muskelzellen. Ihre Frequenz ist in den einzelnen Abschnitten des Magen-Darm-Traktes festgelegt und liegt beim Menschen im Bereich des Magens bei etwa 3 Depolarisationen/ min, im proximalen Dünndarm bei etwa 11-12/min und im unteren Dünndarm bei etwa 8/min. Das Kolon weist ebenfalls langsame Wellen auf, jedoch ist das Muster deutlich unregelmäßiger. Außerdem sind die Kontraktionsfrequenzen der einzelnen Darmabschnitte zwar lokal spezifisch, aber in toto gesehen inhomogen über den Darm verteilt [3],[10] (s. Tab. 1).

Tab. 1: Typische Grundfrequenz der („slow waves“) in den einzelnen Darmregionen beim Menschen.

\begin{tabular}{ll}
\hline Region & Slow-Wave-Frequenz (pro Minute) \\
\hline Magen (Korpus/Antrum) & $3-5$ \\
Proximaler Dünndarm (Duodenum & $10-12$ \\
Distaler Dünndarm (Ileum) & $7-8$ \\
Kolon & $3-6$ (Oszillation) \\
\hline
\end{tabular}


Während man früher von einem rein muskulären Schrittmacher ausging, weiß man heute, dass die „slow waves“ in spezialisierten, nicht muskulären Zellen, den interstitiellen Cajal-Zellen (ICC), erzeugt werden. Diese Depolarisationen werden über enge Zellkontakte (Gap Junctions) durch elektrotonische Kopplung auf die glatten Muskelzellen übertragen [4],[11]. Das bedeutet aber auch, dass Regionen, die unterschiedliche Slow-Wave-Frequenzen aufweisen (z. B. Magen und Duodenum), elektrisch voneinander isoliert sind.

Die Frequenz der „slow waves“ legt die an einem bestimmten Darmabschnitt maximal mögliche Kontraktionsfrequenz fest. Die „slow waves“ alleine induzieren aber noch keine Kontraktion der glatten Muskulatur, sondern sie legen nur den möglichen Grundrhythmus fest. $\mathrm{Ob}$ es $\mathrm{zu}$ diesem Zeitpunkt $\mathrm{zu}$ einer muskulären Kontraktion kommt, wird durch zusätzliche Reizung (nerval, hormonell) der Muskulatur bestimmt. Diese ist notwendig, um das Auftreten von aktionspotenzialähnlichen, schnellen Entladungen („spike potentials“) zu induzieren und damit die Kontraktionen auszulösen. Das Kontraktionsausmaß und die Kontraktionsdauer werden durch die Anzahl und Frequenz der Spikes festgelegt. Die Frequenz der auftretenden Kontraktionen wird jedoch durch die zugrunde liegende elektrische Basalaktivität (,,slow waves“) vorgegeben [12]. Die rhythmischen Depolarisationen der ICC werden durch rhythmische Schwankungen der intrazellulären Kalziumkonzentrationen der ICC-Zellen erzeugt, die wiederum durch spezielle $\mathrm{Ca}^{2+}$-Kanäle und einen $\mathrm{Ca}^{2+}$-aktivierten $\mathrm{Cl}^{-}$Kanal (Ano1) vermittelt werden [13].

Bei den ICC handelt es sich um eine spezialisierte, nicht muskuläre Zellpopulation, die enge Zellkontakte (Gap Junctions) zu den glatten Muskelzellen, aber auch eine enge nachbarschaftliche Beziehung zu den Nervenfasern aufweisen [11],[14]. Die ICC sind keine einheitliche Zellpopulation und es existieren unterschiedliche Formen, denen auch eine unterschiedliche Funktion zugeschrieben wird. Eine Gruppe der ICC bildet ein dichtes Netzwerk in der Nähe des myenterischen Plexus an der Grenzschicht zur Submukosa (ICC-MY), während eine andere Gruppe zwischen den glatten Muskelfasern und an den Nervenendigungen (ICC-IM, ICC-SMP, ICC-MP) lokalisiert ist. Neben der Induktion der „slow waves“ an der glatten Muskelzelle, haben die ICC vermutlich auch die Aufgabe, die neuralen Impulse an die glatte Muskulatur zu vermitteln. Die ICC sind quasi als Relaiszellen zwischen die terminalen Nervenendigungen und die glatten Muskelzellen eingelagert und übertragen sowohl die exzitatorischen wie auch die inhibitorischen Impulse [15],[16].

Durch die Aktivierung von Motoneuronen kommt es zur Freisetzung von exzitatorischen oder inhibitorischen Substanzen, die dann unter Vermittlung der ICC auf die glatte Muskulatur einwirken. Es ist von wichtiger physiologischer Bedeutung, dass nicht nur erregende Transmitter eine Kontraktion auslösen können. Die Darmmuskulatur in den meisten Regionen des Gastrointestinaltraktes steht im Ruhezustand unter einer neural vermittelten tonischen Hemmung. Mit anderen Worten: Die Darmmuskulatur wird kontinuierlich durch die Freisetzung hemmender Neurotransmitter, wie z. B. Stickstoffmonoxid (NO) und vasoaktives intestinales Peptid 
(VIP), vor einer Kontraktion bewahrt. In diesem „Ruhezustand“ kann eine Kontraktion daher entweder durch einen exzitatorischen Mediator oder aber durch Reduktion des hemmenden Tonus, z. B. eine präsynaptische Hemmung an den inhibitorischen Motoneuronen, ausgelöst werden. Dieser Wirkmechanismus ist z. B. für die erregende Wirkung von Opiaten, Motilin oder Alpha-2-Adrenorezeptoragonisten verantwortlich und scheint gerade für eine Reihe von motilitätswirksamen Substanzen einen wichtigen Angriffspunkt und Wirkmechanismus darzustellen [2],[17].

Neben den ICC kommen aber noch weitere interstitielle Zellen im Gastrointestinaltrakt vor, die nicht c-Kit-positiv sind und sich von den IC nach Cajal unterscheiden. Diese interstitiellen Zellen sind PDGFR $\alpha$-(Platelet Derived Growth Factor alpha-) positiv und scheinen an der purinergen Neurotransmission (ATP, ADP, BNAD) über den P2Y1-Rezeptor beteiligt zu sein. Es wurde daher u. a. ein sog. SIP-Synzytium ( $\mathrm{S}=$,smooth muscle cells“, I = interstitielle Zellen nach Cajal, P = PDGRF-positive IC-Zelle) postuliert, das für die intakte Neurotransmission im Gastrointestinaltrakt notwendig ist [18].

In den Sphinkterregionen, wie dem unteren ösophagealen Sphinkter, dem Pylorus, dem Sphinkter Oddi und dem internen analen Sphinkter, scheint dieser basale inhibitorische Tonus vermindert zu sein und auch die Organisation und Dichte der ICC ist verändert. Dies kann z. T. für die myogene Kontraktion in den Sphinkterregionen verantwortlich gemacht werden. Zwar verfügen die Sphinkterregionen über eine ausgeprägte inhibitorische Innervation, diese wird aber erst bei Bedarf, d. h. zur Öffnung des Sphinkters, aktiviert [19],[20],[21]. Sowohl im unteren ösophagealen Sphinkter, im Pylorus, im Sphinkter Oddi und im internen analen Sphinkter wird diese Inhibition vorrangig durch den Neurotrasmitter NO vermittelt [22],[23],[24],[25]. Störungen der NO-Synthase, dem entscheidenden Enzym der NO-Erzeugung, sind mit einem charakteristischen klinischen Phänotyp wie der Achalasie oder der hypertrophen Pylorusstenose verbunden.

\subsection{Muskelkontraktion und Freisetzung von Kalzium}

Für die glattmuskuläre Kontraktion sind wie für die quergestreifte Muskulatur die Freisetzung und der Anstieg des intrazellulären Kalziumspiegels der wesentliche Mechanismus. Der Anstieg des intrazellulären Kalziums wird einerseits durch $\mathrm{Ca}^{2+}$-Einstrom über rezeptor- oder membranpotenzialgesteuerte Kalziumkanäle und anderseits durch intrazelluläre Freisetzung aus membrangebundenen Speichern oder aus dem sarkoplasmatischen Retikulum vermittelt [26]. Die Regulation des intrazellulären $\mathrm{Ca}^{2+}$-Spiegels erfolgt durch ein komplexes Zusammenspiel der Second-Messenger-Systeme.

Für die Erhöhung des intrazellulären $\mathrm{Ca}^{2+}-$ Spiegels nach Stimulation ist der Einstrom von extrazellulärem $\mathrm{Ca}^{2+}$ über „receptor operated calcium channels“ (ROC) oder durch Membrandepolarisation (,voltage operated calcium channels“, VOC) von 
entscheidender Bedeutung. Die Bedeutung der einzelnen Mechanismen unterscheidet sich von der Lokalisation und der Grundaktivität der untersuchten Muskulatur.

Die Funktion der VOC kann auch durch Neurotransmitter, Hormone oder andere Substanzen in ihrer Aktivität beeinflusst werden. Die Bindung eines exzitatorischen Agonisten an seinen Rezeptor führt zu einer Depolarisation der Zellmembran und damit zu einer Öffnung des membranpotenzialabhängigen $\mathrm{Ca}^{2+}$-Kanals (VOC). Außerdem können die Kalziumkanäle über die Aktivierung eines Second-Messenger-Systems, wie z. B. der Adenylatzyklase, beeinflusst werden. Der Anstieg von cAMP führt zur Aktivierung der Proteinkinase A, die ihrerseits zur Phosphorylierung des $\mathrm{Ca}^{2+}-\mathrm{Ka}^{-}$ nals oder eines mit ihm assoziierten Proteins führt. Dies bewirkt eine Konformationsänderung des Kanals, sodass sich dieser bei Membrandepolarisierung mit größerer Wahrscheinlichkeit öffnet als im Ruhezustand.

Im Gegensatz dazu führt bei den ROC die Bindung des Agonisten an seinen Rezeptor direkt zur Öffnung des rezeptorassoziierten Kanals. Die Wiederherstellung des Ruhezustands erfolgt durch einen $\mathrm{Na}^{+} / \mathrm{Ca}^{2+}$-Austausch über die Zellmembran und durch membranständige, ATP-abhängige $\mathrm{Ca}^{2+}$-Pumpen an der Zellmembran, an den Mitochondrien und am sarkoplasmatischen Retikulum.

Der Anteil von extrazellulärem oder intrazellulär freigesetztem $\mathrm{Ca}^{2+}$ an der Erhöhung des zytoplasmatischen $\mathrm{Ca}^{2+}$-Spiegels ist je nach Organ, Spezies und Wandschicht unterschiedlich. Während in der Zirkulärmuskulatur und der Muskulatur in den Sphinkterregionen meist der Anteil der intrazellulären Kalziumspeicher überwiegt, ist in der Longitudinalmuskulatur der Einstrom von extrazellulärem Kalzium von größerer Bedeutung.

\section{Enterisches Nervensystem}

Das enterische Nervensystem besteht aus mehreren ganglienhaltigen Plexus, die untereinander vernetzt sind und Nervenausläufer zur glatten Muskulatur, zu Gefäßen und zur Mukosa des Gastrointestinaltraktes besitzen. Das Verteilungsmuster der intramuralen Plexus ist innerhalb des Gastrointestinaltraktes gleich, variiert aber bezüglich Größe und Form der Ganglien in den verschiedenen Spezies und Regionen [1].

Im Gegensatz zum sympathischen und parasympathischen Nervensystem vermag das enterische Nervensystem nach Ausschaltung zentraler Einflüsse noch relativ komplexe und koordinierte Abläufe auszuführen. Dazu zählen etwa die intestinale Peristaltik mit der Propulsion von Nahrungsbrei sowie sekretorische und vasomotorische Reflexe. Eine Voraussetzung dafür ist, dass im enterischen Nervensystem komplette Reflexbögen mit sensorischen Nerven, Interneuronen sowie exzitatorischen und inhibitorischen Interneuronen vorhanden sind. Dies wiederum kann nur durch funktionell verschiedene Neurone gewährleistet werden, die nach ihrer Morphologie und durch unterschiedliche methodische Ansätze wie Immunhistochemie und Elektrophysiologie subklassifiziert werden können [27],[28]. 
Neben den beiden klassischen Neurotransmittern Noradrenalin und Acetylcholin sind im autonomen Nervensystem inzwischen eine Vielzahl von Neurotransmittern nachgewiesen worden. Neben Substanzen wie Serotonin und Stickstoffmonoxid (NO), Gamma-Aminobuttersäure (GABA) oder Adenosintriphosphat (ATP) gehört da$\mathrm{zu}$ vor allem die große heterogene Gruppe der gastrointestinalen Neuropeptide [28], [29],[30]. Auch ursprünglich klassische Hormone wie Cholezystokinin (CCK) oder Somatostatin wurden im enterischen Nervensystem nachgewiesen und üben eine Funktion als Neurotransmitter im klassischen Sinne aus. Ein Überblick über die postulierten und nachgewiesenen Neurotransmitter im enterischen Nervensystem ist in Tab. 2 wiedergegeben.

Tab. 2: Nachgewiesene und postulierte Neurotransmitter im enterischen Nervensystem. ANF = atrialer natriuretischer Faktor; ANP = atrial natriuretic peptid; ATP = Adenosintriphosphat; CCK = Cholezystokinin; CGRP = calcitonin Gene-related peptid; CNP = C-type natriuretic peptid; $C O=$ carbonmonoxyde-neurotransmitter; $\quad C R F=$ Corticotropin-Releasing-Faktor; $G A B A=$ Gamma-Aminobuttersäure; GRP = Gastrin Releasing Peptide; NO = Stickstoffmonoxid; NPY = neuropeptid; PACAP = Pituitary adenylate cyclene activating peptid; PAF = Plättchenaktivierender Faktor; $\mathrm{PHI}=$ phosphohexoseisamerase; PYY = Peptid YY (Hormon); TRH = Thyrotropin-Releasing-Hormon; VIC = vic-neurotransmitter; VIP = vasoaktives intestinales Peptid; VNP = ventricular natriuretic peptid.

\begin{tabular}{|c|c|c|c|}
\hline Nicht-Peptide & Peptide & $\begin{array}{l}\text { Postulierte Neuro- } \\
\text { transmitter }\end{array}$ & Fortsetzung \\
\hline Adenosin & Angiotensin I + II & ANF, ANP, CNP & Melatonin \\
\hline ATP & Bombesin, GRP & Bradykinin & Prostaglandine \\
\hline Azetylcholin & $\mathrm{CCK}$ & Benzodiazepine & PAF \\
\hline Cannabinoide & CGRP & $\mathrm{CO}$ & Stresscopin \\
\hline GABA & Dynorphin A & CRF & TRH \\
\hline Glutamat & Enkephalin & B-Endorphin & Trypsin \\
\hline NO & Galanin & Endothelin & Tryptase \\
\hline Noradrenalin & Neurokinin A & Endomorphin & VIC \\
\hline \multirow[t]{7}{*}{ Serotonin } & NPY & Guanylin & VNP \\
\hline & PACAP & Uroguanylin & \\
\hline & PHI & Histamin & \\
\hline & PYY & Leptin & \\
\hline & Somatostatin & Neurotensin, & \\
\hline & Substanz P & Neuromedin U & \\
\hline & VIP & Nociceptin & \\
\hline
\end{tabular}




\subsection{Grundlage des peristaltischen Reflexes}

Die Auslösung einer peristaltischen Welle erfordert das Ablaufen eines aszendierenden exzitatorischen und eines deszendierenden inhibitorischen Reflexes. Beide Reflexe werden durch ein afferentes Neuron initiiert. Die Transmittersubstanz der afferenten Impulse dürfte Substanz P, CGRP oder Serotonin (5-HT) sein. Ob der Reflex durch spezifische intrinsische, afferente Neurone oder nach dem Prinzip des Axonreflexes abläuft, kann derzeit noch nicht mit Sicherheit beantwortet werden [31],[32], [33].

Der exzitatorische Reflex setzt sich fort in oral projizierende cholinerge Interneurone (chemischer Code: Chat/SP/Calretinin), die schließlich die exzitatorischen Motoneurone zur zirkulären Muskulatur aktivieren. Als Transmitter für die exzitatorischen Impulse gelten Azetylcholin und Substanz P bzw. Neurokinin A [34].

Der deszendierende inhibitorische Reflex wird via afferente Fasern über anal projizierende Interneurone mit einem cholinergen und einem unbekannten nicht cholinergen, nicht adrenergen Übertragungsmechanismus vermittelt. Nach der chemischen Kodierung scheint es vier verschiedene deszendierende Interneurone zu geben, die entweder Somatostatin, 5-HT, VIP oder VIP/Chat enthalten. Für den inhibitorischen Effekt, der über inhibitorische Motoneurone an der zirkulären Muskulatur ausgelöst wird, ist Stickstoffmonoxid und eine nicht cholinerge, nicht adrenerge Substanz, möglicherweise VIP oder ATP, verantwortlich [35]. Für die sekretomotorischen Neurone zur Mukosa kommen als Transmitter neben Acetylcholin auch mehrere Neuropeptide infrage. Den nicht cholinergen Effekt vermitteln in erster Linie VIP-haltige Neurone, aber auch Substanz-P-haltige Neurone. Beide Neuropeptide stimulieren die Elektrolyt- und Wassersekretion aus dem Dünndarm.

Neben dem peristaltischen Reflex gibt es im Gastrointestinaltrakt eine Reihe von inhibitorischen Reflexen und Reflexen, die in der Regel über die prävertebralen Ganglien (Ganglion coeliacum, Ganglia mesenterica superior et inferior) oder das sympathische Nervensystem verschaltet werden. Diese inhibitorischen Reflexe sind ebenfalls wie die intrinsischen Reflexe des enterischen Nervensystems von einer intakten Innervation durch das zentrale Nervensystem (ZNS) unabhängig. Es wurden enterogastrische, enteroenterische, kologastrische und koloenterische Reflexe beschrieben [36].

Zusätzlich gibt es aber noch exzitatorische und inhibitorische Reflexe, die auf einer Vermittlung durch das ZNS beruhen, wie z. B. der gastrokolische Reflex.

\section{Grundformen der Motilität}

Die Motilität insbesondere des oberen Gastrointestinaltraktes lässt sich in zwei unterschiedliche Zustandsmuster einteilen:

- die Nüchternmotilität oder auch interdigestive Phase,

- die postprandiale Motilität oder auch digestive Phase. 
Zwar bleiben in beiden Zustandsformen die oben beschriebenen Grundmuster identisch, jedoch ist die übergeordnete Koordination der einzelnen Darmabschnitte verändert.

\subsection{Nüchternmotilitåt (interdigestive Phase)}

Die Motilität des nüchternen Zustands (interdigestive Motilität) ist durch ein sich regelmäßig wiederholendes Grundmuster charakterisiert, das auch Nüchternzyklus genannt wird. Es folgen aufeinander:

- $\quad$ eine Phase der relativen motorischen Ruhe (Phase I),

- $\quad$ eine Phase mit unkoordinierter Aktivität (Phase II),

- eine kurzzeitige Phase regelmäßiger, kräftiger, nach aboral propagierter Kontraktionen (Phase III).

Die Gesamtdauer des motorischen Nüchternzyklus liegt beim Menschen meistens bei 100-120 min, wobei eine erhebliche Schwankungsbreite besteht, die auch noch Zykluslängen von weniger als 60 min oder mehr als $3 \mathrm{~h}$ als normal definiert. Dieses Muster zyklischer Motilität ist sowohl im Magen als auch im gesamten Dünndarm zu beobachten. Den zyklischen motorischen Phänomenen folgen in ihrer Periodik die Änderungen der Sekretionsleistung der exogenen Drüsen (Pankreassekretion, Gallefluss) und die der endokrinen Organe [37].

Die Phase III beginnt in der Regel im Magenantrum und wandert von hier nach distal. Mit entsprechenden Untersuchungsmethoden lassen sich aber auch zyklische Motilitätsveränderungen im Fundus und im unteren Bereich der Speiseröhre nachweisen. Die Phasen I und II sind die beiden zeitlich bestimmenden Phasen, die gemeinsam ca. 90 \% der gesamten Zyklusdauer einnehmen. Ihr relativer Anteil variiert allerdings beim Menschen in der Wach- und der Schlafphase. Die Bezeichnung dieser periodischen Nüchternmotilität ist z. T. etwas verwirrend. Während sich aus der ursprünglichen, elektromyografischen Messung der Aktivitätsspikes in Tiermodellen der Terminus „Migrierender Myoelektrischer (C)Komplex, MMC“ etablierte, wurde bei den humanexperimentellen Untersuchungen diese Abkürzung für „Migrierender Motor Complex“ verwendet, da hier die Aufzeichnung mittels intraluminaler Drucksonden erfolgte [37], [10],[38].

\subsubsection{Phase I}

In dieser Phase sind die elektromyografisch gemessenen „slow waves“ nicht mit entsprechenden Spike-Potenzialen und damit Kontraktionen verknüpft. Die Phase der motorischen Inaktivität ist beim wachen Menschen im Vergleich $\mathrm{zu}$ anderen Spezies relativ kurz und umfasst in der Regel zwischen 5 und 20 \% der Zyklusdauer. Während der Schlafphase ist die Phase I zeitlich gesehen die dominierende Phase und nimmt bis auf 80 \% der Gesamtzyklusdauer zu. Wie bereits erwähnt, besteht bei der Phase I nicht nur motorische, sondern auch sekretorische Inaktivität: Die Magense- 
kretion ist minimal und der Gasfluss ins Duodenum sowie die Pankreassekretion sind reduziert oder eingestellt.

\subsubsection{Phase II}

Phase II ist gekennzeichnet durch unregelmäßig auftretende Kontraktionen von wechselnder Stärke und Frequenz. Von der Form her ist das Kontraktionsmuster der Phase II mit dem digestiven Motilitätsmuster vergleichbar. In dieser Phase sind ca. 40-50 \% aller „slow waves“ mit dem Auftreten entsprechender Spike-Potenziale verbunden. Circa $50 \%$ der auftretenden Kontraktionen sind segmental, die restlichen Kontraktionen sind fortgeleitet, wenn auch größtenteils (ca. 2/3) nur über eine kurze Strecke $(<10 \mathrm{~cm})$.

Beim wachen Menschen ist Phase II die dominante Aktivität des Zyklus, die bis $80 \%$ der periodischen Aktivität beträgt. Während des Schlafs nimmt der prozentuale Anteil der Phase II entsprechend der Zunahme der Phase I deutlich ab und ist oft nur kurz nachweisbar. Parallel zur Zunahme der motorischen Funktionen kommt es auch $\mathrm{zu}$ einer leichten Steigerung der endokrinen und exokrinen sekretorischen Funktionen.

\subsubsection{Phase III}

Die Phase III, oder auch Aktivitätsfront genannt, ist gekennzeichnet durch regelmäßige, kräftige Kontraktionen, die über eine Dauer von 5-10 min in der für die entsprechende Region maximal möglichen Frequenz auftreten. Fast alle „slow waves“ sind von entsprechenden Spike-Potenzialen begleitet und die Kontraktionen werden über größere Strecken (mehr als $50 \%$ der Kontraktionen $>30 \mathrm{~cm}$ ) fortgeleitet. Entsprechend der Frequenz der Slow-Wave-Aktivität handelt es sich um ca. 3 Kontraktionen/min im distalen Magen, 11-12 Kontraktionen/min im proximalen Dünndarm und etwa 8-9 Kontraktionen/min im distalen Dünndarm. Der Ausgangspunkt liegt in der Regel im Magen, die Phase III kann jedoch auch im oberen Dünndarm beginnen. Die Aktivitätsfront der Phase III wandert langsam von oral nach aboral bis ins terminale Ileum mit einer Fortleitungsgeschwindigkeit von $2-4 \mathrm{~cm} / \mathrm{min}$. Die Fortleitungsgeschwindigkeit der Aktivitätsfront, d. h. des Bereiches mit einer gesteigerten Darmmotilität, ist dabei nicht $\mathrm{zu}$ verwechseln mit der Fortleitungsgeschwindigkeit der „slow waves“ und damit der einzelnen Kontraktionen innerhalb dieser Aktivitätsfront, die deutlich höher liegt. Beide Phänomene stellen die Ausprägung unterschiedlicher motorischer Phänomene dar, die in einem unterschiedlichen Zeitraster ablaufen.

Die regelhafte Fortleitung und Ausbreitung der Aktivitätsfront der Phase III ist von der Intaktheit des enterischen Nervensystems abhängig. Außerdem gibt es Hinweise für eine cholinerge Beteiligung bei der Ausbildung der Aktivitätsfront. In einer ausgeschalteten innervierten Dünndarmschlinge findet man zwar auch eine periodische Aktivität, diese weist aber eine höhere Frequenz als der Restdarm auf und der 
zeitliche Ablauf der Aktivität ist nicht mehr mit der des Restdarms koordiniert. Früher wurde insbesondere das Hormon Motilin mit der Auslösung und der Fortleitung der Phase III des MMC in Verbindung gebracht. Motilin zeigt periodische Schwankungen der Plasmaspiegel, die mit den einzelnen MMC-Phasen korrelieren [39]. Dabei findet man während der duodenalen Passage der Phase III einen Peak der Motilinplasmakonzentration. Vermutlich ist Motilin ein Verstärkermechanismus, der die Ausbildung insbesondere der gastralen Phase III mit begünstigt und deren Fortleitung unterstützt. Als alleiniger auslösender Mechanismus der Phase-III-Aktivität wird es heute jedoch nicht mehr angesehen. Mit dem Aktivitätspeak in Phase III kommt es parallel zu einer deutlichen Stimulation der Sekretion der Magensäure sowie der Pankreas- und Gallesekretion.

\subsubsection{Physiologische Rolle der Nüchternmotilität}

Die physiologische Rolle der Nüchternmotilität war Anlass vieler Spekulationen. Am verbreitetsten ist das Konzept der „Reinigungsfunktion“ („housekeeper“) [40]. Durch die starken propulsiven Kontraktionen und die begleitende Stimulation der Sekretion von Magen und Pankreas kommt es zu einer kompletten Entleerung des Magens und des Dünndarms von Fremdkörpern oder unverdaulichen Nahrungsbestandteilen. Dieser mechanischen Reinigung wird eine wichtige Rolle bei der Darmhomöostase zugeschrieben. Störungen dieser Funktion führen zur Stase und Retention von Nahrungsbestandteilen und begünstigen die Fehlbesiedelung von Dünndarmabschnitten mit Keimen oder die Bildung von Bezoaren [41]. Insbesondere für die Magenentleerung ist das Auftreten der Phase III im Antrum eine entscheidende Komponente. Durch die Siebfunktion des Pylorus werden postprandial nur zerkleinerte Nahrungsbestandteile von < $1 \mathrm{~mm}$ aus dem Magen entleert. Größere Nahrungsreste und nicht zerkleinerbare Bestandteile (Faserstoffe, Fremdkörper, säureresistente Tabletten) können in dieser Phase den Magen nicht verlassen, sondern bleiben hier zunächst liegen. Mit dem Auftreten der ersten Phase-III-Aktivität mit antraler Komponente werden diese unverdaulichen Bestandteile aus dem Magen entleert und in der Regel auch schnell weiter nach distal transportiert [42]. Der Nachweis der funktionellen Bedeutung dieses Mechanismus gelang u. a. sehr elegant durch die Erfassung der Magenentleerung mit einer Metallkugel mittels eines Metalldetektors.

\subsection{Postprandiale Motilitåt (digestive Phase)}

Kommt es zur Nahrungsaufnahme, so wird das interdigestive Motilitätsmuster unmittelbar unterbrochen und durch ein gleichförmiges Motilitätsmuster ersetzt, das Kontraktionen von unterschiedlicher Frequenz und Amplitude aufweist. Diese Kontraktionen sind teilweise stationär und dienen der Durchmischung des Speisebreis mit den Verdauungssäften, teilweise sind die Kontraktionen fortgeleitet und dienen der Propulsion des Speisebreis. Die Fortleitung erfolgt aber im Gegensatz zur Phase-IIIAktivität der interdigestiven Motilität meist nur über kürzere Distanzen [43]. 
Die Kontraktionen in der digestiven Phase ähneln in ihrer Zusammensetzung aus segmentalen und fortgeleiteten Kontraktionen der Phase II der interdigestiven Motilität, jedoch treten die Kontraktionen z. T. in sog. Clustern, d. h. gruppierten Aktivitätsphasen auf, die von längeren Ruhephasen gefolgt sind.

Der Wechsel von interdigestiver zu digestiver Motilität setzt abrupt und entlang des gesamten Darmes ein. Eine ausgeschaltete, aber noch innervierte Darmschlinge zeigt ebenfalls einen regelrechten Wechsel von Nüchternmotilität auf postprandiale Motilität, während dies bei einer extrinsisch denervierten Schlinge nicht mehr der Fall ist. Es konnte $u$. a. gezeigt werden, dass eine intermittierende, durch Kühlung hervorgerufene Vagusblockade das digestive Motilitätsmuster unterbricht und dass das interdigestive Muster wieder zum Vorschein kommt. Dies bedeutet, dass die Umschaltung von interdigestiver auf digestive Motilität auf eine intakte extrinsische Innervation, insbesondere durch den N. vagus, angewiesen ist. Die digestive Motilität ist vermutlich auf eine gesteigerte extrinsische vagale Aktivität zurückzuführen.

Andererseits können auch einige exogen zugeführte Hormone, wie Gastrin oder CCK, aber auch pankreatisches Polypeptid, Insulin oder Sekretin, eine Umschaltung von interdigestiver auf digestive Motorik bewirken. Tierexperimentelle Untersuchungen haben gezeigt, dass insbesondere CCK auf vagale Afferenzen einwirken und vermutlich so die Motilitätsveränderungen induzieren kann.

Die Dauer einer digestiven Phase ist im Wesentlichen abhängig von der Art, der Zusammensetzung und dem Kaloriengehalt der zugeführten Nahrung [44]. Auch eine Scheinfütterung kann ein digestives Muster der Motilität auslösen, das allerdings nur während der Scheinfütterung anhält. Unter einer Scheinfütterung versteht man das Zeigen oder Kauen der Nahrung, ohne dass die Nahrung geschluckt wird. Auch Magendehnung und die Anwesenheit von Nahrungsstoffen im Darmlumen sind potente Faktoren, die das Nüchternmuster unterbrechen können.

\subsection{Regionale Besonderheiten}

Neben dieser generellen Umstellung der Motilitätsmuster durch Nahrungsaufnahme kommt es in den einzelnen Abschnitten des Magen-Darm-Traktes zu spezifischen Veränderungen der Motilität. Diese werden in den einzelnen Abschnitten zu den Organsystemen (Ösophagus, Magen, Dünndarm, Kolon, Anorektum) besprochen und dargelegt. 


\section{Literatur}

[1] Furness JB, Costa M. The enteric nervous system. Edinburgh, London: Churchill Livingstone 1987.

[2] Wood JD. Excitation of intestinal muscle by atropine, tetrodotoxin and xylocaine. Am J Physiol 1972; 222: 118-125.

[3] Alvarez WC, Mahoney LJ. Action currents in stomach and intestine. Am J Physiol 1922; 58: 476493.

[4] Thuneberg L, Rumessen JJ, Mikkelsen HB. The interstitial cells of Cajal: Intestinal pacemaker cells? In: Wienbeck M (ed). Motility of the Digestive Tract. New York: Raven Press 1982; 115-122.

[5] Bayliss WM, Starling EH. The movements and innervation of the small intestine. J Physiol 1899; 24: 99-143.

[6] Trendelenburg P. Physiologische und pharmakologische Versuche über die Dünndarmperistaltik. Naunyn Schmiedeberg's Arch Pharmacol 1917; 81: 55-129.

[7] Szurszewski JH. A migrating electrical motor complex of the canine small intestine. Am J Physiol 1969; 217: 1757-1763.

[8] Code CF, Marlett JA. The interdigestive myoelectric complex of the small bowel of dogs. J Physiol 1975; 246: 289-309.

[9] Burnstock G, Costa M. Inhibitory innervation of the gut. Gastrenterology 1973; 64: 141-144.

[10] Szurszeswski JH. A migrating electric complex of the canine small intestine. Am J Physiol 1969; 217: $1757-1763$.

[11] Rumessen JJ, Thuneberg L. Pacemaker cells in the gastrointestinal tract: interstitial cells of Cajal. Scand J Gastroenterol Suppl 1996; 216: 82-94.

[12] Sarna SK, Daniel EE. Electrical stimulation of gastric electrical control activity. Am J Physiol 1973; 255: 125-131.

[13] Singh RD, Gibbons SJ, Saravanaperumal SA et al. Ano1, a Ca2+ -activated Cl- channel, coordinates contractility in mouse intestine by $\mathrm{Ca} 2+$ transient coordination between interstitial cells of Cajal. J Physiol 2014; 592: 4051-4068.

[14] Publicover NG, Horowitz NN, Sanders KM. Calcium oscillations in freshly dispersed and cultured interstitial cells from canine colon. Am J Physiol 1992; 262: C589-C597.

[15] Salmhofer H, Neuhuber WL, Ruth P et al. Pivotal role of the interstitial cells of Cajal in the nitric oxide signaling pathway of rat small intestine. Morphological evidence. Cell Tissue Res 2001; 305: 331-340.

[16] Klein S, Seidler B, Kettenberger A et al. Interstitial cells of Cajal integrate excitatory and inhibitory neurotransmission with intestinal slow-wave activity. Nat Commun 2013; 4: 1630.

[17] Manaka H, Manaka Y, Kostolanska F et al. Release of VIP and substance P from isolated perfused canine ileum. Am J Physiol 1989; 257: G182-G190.

[18] Sanders KM, Koh SD, Ro S, Ward SM. Regulation of gastrointestinal motility-insights from smooth muscle biology. Nat Rev Gastroenterol Hepatol 2012; 9: 633-645.

[19] Allescher HD, Daniel EE, Dent J et al. Extrinsic and intrinsic neural control of pyloric sphincter pressure in the dog. J Physiol 1988; 401: 17-34.

[20] Allescher HD. Papilla of Vater: structure and function. Endoscopy 1989; 21 (Suppl 1): 324-329.

[21] Allescher HD. Pylorus: Myogenic and neural control of the pyloric sphincter. In: Daniel EE, Tomita T, Tsuchida S, Watanabe M (eds). Sphincters: Normal Function - Changes in Diseases. Boca Raton: CRC-Press 1992; 83-115.

[22] Bult H, Boeckxstaens GE, Pelckmans PA et al. Nitric oxide as an inhibitory non-adrenergic noncholinergic neurotransmitter. Nature 1990; 345: 346-347.

[23] Boeckxstaens GE, Pelckmans PA. Nitric oxide and the NANC neurotransmission. Comp Biochem Physiol 1997; 118a: 925-937. 
[24] Allescher HD, Tougas G, Vergara P et al. Nitric oxide as a putative non-adrenergic non-cholinergic inhibitory transmitter in the canine pylorus in vivo. Am J Physiol 1992; 262: G695-G702.

[25] Allescher HD, Lu S, Daniel EE, Classen M. Nitric oxide as putative nonadrenergic noncholinergic inhibitory transmitter in the opossum sphincter of Oddi. Can J Physiol Pharmacol 1993; 71: 525530.

[26] van Breemen C, Chen Q, Laher I. Superficial buffer barrier function of smooth muscle sarcoplasmic reticulum. Trends Pharmacol Sci 1995; 16: 98-105.

[27] Costa M, Furness JB. The peristaltic reflex: an analysis of the nerve pathways and their pharmacology. Arch Pharmacol 1976; 294: 47-60.

[28] Costa M, Furness JB, Llewellyn-Smith IJ: Histochemistry of the enteric nervous system. In: Johnson LR (ed). Physiology of the gastrointestinal tract. New York: Raven Press 1987; 1-40.

[29] Costa M, Furness JB. Peptidergic nerves in the gut. Br Med Bull 1982; 38: 247-252.

[30] Allescher HD, Ahmad S. Postulated physiological and pathophysiological roles on motility. In: Daniel EE (ed). Neuropeptide function in the gastrointestinal tract. Boca Raton: CRC Press 1990; 309-400.

[31] Grider JR, Jin JG. Intestinal peristalsis is regulated by a sensory axon reflex with neuronal cell body in the dorsal root ganglion. Gastroenterology 1991; 100: 927.

[32] Smith TK, Bornstein JC, Furness JB. Convergence of reflex pathways excited by distension and mechanical stimulation of the mucosa onto the same myenteric neurons of the guinea pig small intestine. J Neurosci 1992; 12: 1502-1510.

[33] Grider JR, Kuemmerele JF, Jin JG. 5-HT released by mucosal stimuli initiates peristalsis by activating 5-HT4 / 5-HT1p receptors on sensory CGRP neurons. Am J Physiol 1996; 270: 778-782.

[34] Bartho L, Holzer P, Donnerer J, Lembeck F. Evidence for the involvement of substance $P$ in the atropine resistant peristalsis of the guinea pig ileum. Neurosci Lett 1982; 32: 69-74.

[35] Grider JR. Interplay of VIP and nitric oxide in regulation of the descending relaxation phase of peristalsis. Am J Physiol 1993; 264: G334-G340.

[36] Kreulen DL, Szurszewski JH. Reflex pathways in the abdominal prevertebral ganglia: Evidence for a colo-colonic inhibitory reflex. J Physiol (Lond) 1979; 295: 21-32.

[37] Rees WD, Malagelada JR, Miller LJ, Go VLW. Human interdigestive and postprandial gastrointestinal motor and gastrointestinal hormone patterns. Dig Dis Sci 1982; 27: 321-329.

[38] Daniel EE, Fox JET, Collins SM et al. Initiation of migrating myoelectric complexes in human subjects: Role of duodenal acidification and plasma motilin. Can J Physiol Pharmacol 1981; 59: 173-179.

[39] Vantrappen G, Janssens J, Peeters TL et al. Motilin and interdigestive motor complex in man. Dig Dis Sci 1979; 24: 497-500.

[40] Code CF, Schlegel JF. The gastrointestinal housekeeper. In: Daniel EE (ed). Gastrointestinal Motility. Vancouver: Mitchell Press 1974; 631-633.

[41] Vantrappen G, Janssens J, Ghoos Y. The interdigestive motor complex of normal subject and patients with bacterial overgrowth of the small intestine. J Clin Invest 1977; 59: 1158-1166.

[42] Weisbrodt NW, Wiley JN, Overholt BF, Bass P. A relation between gastroduodenal muscle contractions and gastric emptying. Gut 1969; 10: 543-548.

[43] Ehrlein HJ, Schmid HR, Feinle C. Characteristic motor patterns of phase II and behaviour of phase III in the fed state. J Gastrointest Mot 1992; 4: 317-327.

[44] Lin HC, Doty JE, Reedy TJ, Meyer JH. Inhibition of gastric emptying by sodium oleate depends on length of intestine exposed to nutrient. Am J Physiol 1990; 259: G1031-G1036. 
